\title{
TOTAL LUNG CAPACTTY, RESIDUAL VOLUME AND PREDICTED RESIDUAL VOLUME IN A DENSITOMETRIC STUDY OF OLDER MEN
}

\author{
R. W. LATIN, PhD and R. O. RUHLING, PhD, FACSM
}

Human Performance Research Laboratory, College of Health, The University of Utah, Salt Lake City, Utah 84112

\section{ABSTRACT}

Results of investigations using various lung volumes for hydrostatic weighing determinations (HWD) appear to be inconclusive. Often, these lung volumes are predicted and not clinically determined. For this reason, total lung capacity (TLC), a measured residual volume (RV), and a predicted residual volume (PRV) were used during HWDs to compare the techniques. Twenty-five older men, 56 to 70 years $(\bar{X} \pm 62.1+4.2$ yrs) performed HWDs at RV (10 trials) and at TLC (3-5 trials). Values for body density and fat free mass were not significantly different between RV and TLC; both values were, however, significantly different from those derived using PRV. There were statistically significant differences $(p<0.05)$ between all 3 per cent body fat values but the 1.1 per cent difference between TLC and RV may not be physiologically important. It was concluded that TLC and RV may be used comparably during HWDs, but a PRV may produce significantly different values. Since HWD at TLC is easily performed and circumvents the difficulties associated with the RV technique, it may be the preferred method for older subjects.

\section{INTRODUCTION}

While a correction for residual volume (RV) is necessary for the most accurate determination of body density (Db), investigators have examined the efficacy of other lung volumes and capacities for use in human densitometry. Welch and Crisp (1958) explored the reproducibility of $\mathrm{Db}$ measurements following a maximum expiration and approximately a one-half maximum expiration. They reported the average density following maximum expiration was significantly lower than that determined at maximum expiration. Conversely, Thomas and Etheridge (1980) reported no statistically significant difference in Db measurements assessed at RV and functional residual capacity (FRC). Due to this relationship they concluded, that hydrostatic weighing (HW) at FRC may be the method of choice since it is more comfortable for the subject. Weltman and Katch (1981) examined body density measurements as determined by HW at RV and TLC, from 72 male and 51 female middle-aged subjects. Statistically significant differences, between measures of Db, at RV and TLC, were observed. However, per cent body fat (PBF) values for the RV and TLC data differed by only $0.5 \%$ for men and $0.9 \%$ for women. Since these results indicated that negligible differences existed between PBF determinations at RV and TLC, the authors suggested that the latter may be the method of choice for subjects uncomfortable with performing the technique at RV.

Timson and Coffman (1984) also established $\mathrm{Db}$ and PBF measures from 50 male and 50 female middle-aged subjects obtained from HWs of RV (on-land) and TLC (onland and in-water). They reported statistically significant differences for Db and PBF values obtained at $R V$ and TLC (on-land). However, no significant difference was observed between these measures when obtained by $\mathrm{HW}$ at RV and TLC (in-water). These authors also reported the TLC procedure to be less difficult for the subjects to perform.

Address for corrspondence:

Richard W. Latin

School of HPER

University of Nebraska at Omaha

Omaha

NE 68182
Often, RVs are predicted, rather than being clinically determined. Wilmore (1969) examined the differences of $D b$ as assessed by HW at actual, predicted, and constant RVs in 69 male and 128 female college-aged subjects. Although no statistical differences were observed among any of the methods. Wilmore specified that enough individual variation existed among these methods to warrant the use of actual RV when absolute accuracy is essential, e.g., research studies. However for purposes of classification, diagnosis, screening, or similar procedures it would appear unnecessary to obtain an absolutely accurate measure of RV. Moreover, since RV is subject to considerable variability with such factors as age, height, and sex, care should be taken in the selection of an appropriate prediction method for this lung volume (Brozek, 1960).

Since previous investigations suggest that HW at larger lung volumes are more comfortable for the subject and provide very stable scale readings, we sought to compare body composition parameters generated from HW at TLC and RV, in a population of individuals that may have difficulty performing the RV manoeuvre. In addition, body composition parameters generated from a PRV were compared with the other two lung volume techniques.

\section{Methods}

Prior to the initiation of this study, approval from the University of Utah Review Committee for Research with Human Subjects (Health Sciences) was obtained. As required by the Review Committee, an informed consent was obtained in writing, from all subjects. All testing procedures were thoroughly explained to each subject. The subjects were also informed that they could withdraw from the study at any time without prejudice.

\section{Subject selection}

Twenty-five volunteer subjects were selected for this study. The subjects were Caucasian males, 56 to 70 years of age.

All subjects were free from known respiratory disorders which impair normal lung function (Crofton and Douglas, 1975) or conditions which may have indicated dehydration or hyperhydration (Girandola et al, 1977). This was established to assure a physiologically valid measure of each subject's body composition. Furthermore, subjects 
were required to complete a medical history questionnaire. The physical characteristics of the subjects are presented in Table I.

TABLEI

Physical characteristics of subjects $(n=25)$

\begin{tabular}{lrr}
\hline Variable & Mean & \pm S.D. \\
\hline Age (yr) & 62.11 & \pm 4.17 \\
Height (cm) & 176.24 & \pm 6.17 \\
Weight (kg) & 87.69 & \pm 9.95 \\
Total lung capacity (1) & 7.37 & \pm 1.04 \\
Residual volume (1) & 2.64 & \pm 0.41 \\
Predicted residual volume (1) & 1.82 & \pm 0.10 \\
\hline
\end{tabular}

\section{Height and weight}

Height was measured to the nearest $\mathrm{cm}$ by using a medical grade stadiometer with the subject standing barefoot, his feet together, and his head level. Weight was determined with the subject in athletic shorts on a balance beam platform scale to the nearest $0.1 \mathrm{~kg}$. In addition, age was reported in years.

\section{Hydrostatic weighing}

Hydrostatic weighing was conducted according to the technique described by Katch et al (1967). A PVC pipe platform was suspended in a shallow, fresh water swimming pool from a $9 \mathrm{~kg}$ Chatillon autopsy scale (accurate to $10 \mathrm{~g}$ ). Ten trials were made after a maximal expiratory effort (RV) with an average of the last three trials used as the "true" underwater weight (Katch, 1968, 1969). The subjects were then instructed to inhale maximally while three to five trials were then made at TLC with an average of the last two trials used to compute the underwater weight (Weltman and Katch, 1981). This sequence was used for testing all of the subjects. To minimise platform movement and to counteract the added buoyancy at TLC, a $5 \mathrm{~kg}$ diver's weight belt was attached to the subject's waist. The belt was positioned in a manner so that chest expansion was not inhibited.

Body density (Db) was calculated using the formula of Brozek et al (1963). The per cent body fat (PBF) was derived according to the Siri formula (1956). Separate Db, PBF, and FFM values were derived from RV, TLC, and PRV data.

\section{Lung volumes}

Prior to hydrostatic weighing determinations each subject had assessments of RV and vital capacity (VC) made. Residual volume was determined, in the laboratory with the subjects in a seated position, by the single-breath helium dilution technique using a Collins P2100 helium residual volume unit. Vital capacity (VC) was determined in a similar position, by using a Collins $13.5 \mathrm{~L}$ wet spirometer. These techniques were repeated until no more than $100 \mathrm{ml}$ difference existed between duplicate trials. The average of the two trials was used as the measure for RV and VC. Total lung capacity was computed by summing vital capacity with RV. Predicted RV was derived by means of the equation reported by Boren et al (1966). This equation is:

Predicted RV $=0.019 \mathrm{Hcm}+0.0155 \mathrm{~A}-2.24$ in which

$\mathrm{Hcm}=$ height in $\mathrm{cm}$ and $A=$ age in years.

This prediction is applicable to all adult males who are asymptomatic with regard to respiratory function. Although several methods for the prediction of RV are reported, we selected Boren et al (1966) for comparison based on its wide applicability and associated statistical accuracy.

\section{Results}

Hydrostatic weight scores were generated by weighing trials at a measured RV and TLC. These scores were used to derive values of $\mathrm{Db}, \mathrm{PBF}$, and FFM, with the means and standard deviations of these variables depicted in Table II. The variables were examined using a one-way ANOVA with repeated measures to detect if significant differences existed among the group means. All of the ANOVAs yielded significant $F$ ratios $(p<0.05)$ which are reported in Table III. To determine which means differed significantly from each other, a Newman-Keuls Multiple Range Comparison Test was applied to these groups. These comparisons also appear in Table III. Correlation coefficients for all experimental variables are reported in Table IV. Values of Db and FMM at RV and TLC exhibited significant differences with PRV. Significant differences among all lung volumes were observed among the PBF variables.

TABLE \|

Statiotical analyais of body density, per cent body fat, and fat free mass data (mean \pm S.D., $n=25$ )

\begin{tabular}{lrrr}
\hline & $\begin{array}{r}\text { Predicted } \\
\text { Residual } \\
\text { Volume }\end{array}$ & \multicolumn{1}{c}{$\begin{array}{l}\text { Residual } \\
\text { Volume }\end{array}$} & \multicolumn{1}{c}{$\begin{array}{r}\text { Total Lung } \\
\text { Capacity }\end{array}$} \\
\hline Density $\left(\mathrm{kg} \cdot \mathrm{m}^{-3}\right)$ & $1037.8 \pm 9.2$ & $1047.4 \pm 8.5$ & $1049.3 \pm 9.0$ \\
Fat Free Mass $(\mathrm{kg})$ & $63.6 \pm 6.5$ & $67.7 \pm 6.5$ & $68.4 \pm 6.9$ \\
Per cent Body Fat $(\%)$ & $27.3 \pm 4.3$ & $22.9 \pm 4.1$ & $21.8 \pm 4.1$ \\
\hline
\end{tabular}

TABLE III

Summary of one way ANOVA's with repeated measures and Newman-Keuls Multiple Range Comparison Tests

\begin{tabular}{lcccc}
\hline $\begin{array}{l}\text { Dependent } \\
\text { Variable }\end{array}$ & $\begin{array}{c}\text { ANOVA } \\
\text { F2,48 }\end{array}$ & $\begin{array}{c}\text { Predicted Residual } \\
\text { Volume }\end{array}$ & $\begin{array}{c}\text { Residual } \\
\text { Volume }\end{array}$ & $\begin{array}{c}\text { Total Lung } \\
\text { Capacity }\end{array}$ \\
\hline $\mathrm{Db}\left(\mathrm{kg} \cdot \mathrm{m}^{-3}\right)$ & $60.2^{*}$ & 1037.8 & 1047.4 & 1049.3 \\
FFM $(\mathrm{kg})$ & $77.1^{*}$ & 63.6 & 67.7 & 68.4 \\
\hline PBF $(\%)$ & $65.9^{*}$ & 27.3 & 22.9 & 21.8 \\
\hline
\end{tabular}

* $p<0.05$

no significant difference between groups $(p>0.05)$

TABLE IV

Pearson Product moment correlation coefficionts among experimental veriables $(n=25)$

\begin{tabular}{lcllll}
\hline & $\begin{array}{c}\text { Lung Volumes } \\
\text { RV }\end{array}$ & PRV & & \multicolumn{1}{c}{ Db } & \\
PRV & 0.32 & $*$ & & RV & PRV \\
TLC & 0.56 & 0.27 & PRV & 0.84 & $*$ \\
& PBF & & TLC & 0.93 & 0.73 \\
& RV & PRV & & FFW & \\
PRV & 0.84 & $*$ & & RV & PRV \\
TLC & 0.93 & 0.73 & PRV & 0.95 & $*$ \\
& & & TLC & 0.99 & 0.92 \\
\hline
\end{tabular}

$p<0.05=0.396$

$p<0.01=0.505$ 


\section{Discussion}

The results of this investigation demonstrated that body composition parameters determined by hydrostatic weighing at either RV or TLC compared favourably. Only the parameter PBF was significantly different $(p<0.05)$ between the two lung volumes. However, the observed mean difference of PBF at these two volumes was only $1.1 \%$, which is for practical purposes, not physiologically significant. In support of this, Weltman and Katch (1981) described significant differences among body composition parameters obtained from RV and TLC with negligible physiological differences.

Timson and Coffman (1984) also reported negligible PBF differences between RV and TLC (in-water) for their male and female subjects $(0.3 \%$ and $0.1 \%$ respectively). They also determined that significant differences existed between PBF values as determined by RV and TLC (on-land) with male and female differences of $2.0 \%$ and $1.4 \%$, respectively. These were greater than the respective $0.5 \%$ and $0.9 \%$ reported by Weltman and Katch (1981). Timson and Coffman explained the importance of establishing a measure of TLC while the subject is submerged in water to take into account the compressing effect that hydrostatic pressure has on TLC, VC, and other lung volumes. Although the subjects in this study had on-land determinations of TLC and demonstrated a $1.1 \%$ difference, one may suspect that this margin of error may be reduced if in-water determinations of TLC are made, thus increasing the accuracy of this technique for this age group.

Significant differences were depicted among all body composition parameters obtained when using hydrostatic weighing at PRV (Table III). This variation may be explained by a consistently lower prediction of the subjects's RV by the prediction equation of Boren et al (1966). Although this equation is applicable to adult males, and is based on age and height, it may not be sufficiently sensitive to the individual variation and increasing RV in older populations (Brozek, 1960). This is further supported by the low, nonsignificant correlation ( $r=0.32$ ) between RV and PRV. Moreover these discrepancies emphasise the importance of measuring, rather than predicting the RVs of this population. Residual volumes that are predicted to be smaller that the actual RV result in an underestimation of $\mathrm{Db}$, and hence an overestimation of PBF and underestimation of FFM. It may be feasible, however, that other RV prediction schemes may more accurately estimate the RV of this population. Further examination into this issue may be warranted.

The use of TLC has several practical advantages over RV. The older or untrained subject appears to be more comfortable with hydrostatic weighings at TLC than at RV. The TLC method allows for very stable scale readings which, with the increased immersion time, enables the observer to obtain more accurate measures of underwater weight. Also, those subjects who are apprehensive or fearful of the water are more co-operative when asked to perform the TLC technique. Even subjects who cannot achieve a maximum expiratory effort underwater to allow measurement at RV, easily respond to the TLC procedure. In support of this Timson and Coffman (1984) reported a significant difference between Borg Perceived Exertion scores obtained after performing RV and TLC procedures. The male subject mean scores were 4.0 and 1.8 for RV and TLC methods, respectively where similar female subject scores were 5.0 and 1.7, respectively. (Borg scores of 2 = easy, $\mathbf{3}=$ moderate, $\mathbf{4}=$ somewhat hard, and $\mathbf{5}=$ hard.) Therefore, the TLC method would provide a pragmatic solution for those difficult HW situations, by providing a technique that will yield results not vitally different than the traditional RV method. Furthermore, since no learning effect is associated with the TLC method, 3 to 5 trials are sufficient to produce reliable assessments, thus reducing the amount of time required for measurement (Weltman and Katch, 1981).

In conclusion, the technique of TLC appears to be comparable with the traditional RV procedures, while both appear more accurate than predicting the RV of older men. However, the merits of HW at TLC indicate that it may be the method of choice for older men and its application to other populations should be examined.

\section{References}

Boren, H., Kory, R. and Syner, J., 1966 "The lung volume and its subdivisions in normal men". Am.J.Med. 41: 96-114.

Brozek, J., 1960 "Age difference in residual volume and vital capacity of normal individuals". J.Geront. 15: 115-160.

Brozek, J., Grande, F., Anderson, J. and Keys, A., 1963 "Densitometric analysis of body composition: revision of some quantitative assumptions". NY Acad.Sci. 110 (I): 113-140.

Crofton, J. and Douglas, A., 1975. Respiratory Diseases. Philadelphia J. B. Lippincott Co.

Girandola, R., Wisewell, R. and Romero, G., 1977 “Body composition changes resulting from fluid ingestion and dehydration". Research Quarterly for Exercise \& Sport 48: 299-303.

Katch, F., 1968 "Apparent body density and variability during underwater weighing". Research Quarterly for Exercise \& Sport 39: 993-999.

Katch, F. 1969 "Practice curves and errors of measurement in estimating underwater weight by hydrostatic weighing". Med.Sci.Sports 1: 212-216.

Katch, F., Michael, E. and Horvath, S., 1967 "Estimation of body volume by underwater weighing: description of a simple method". J.Appl.Physiol. 23: 811-813.

Siri, W., 1956. Gross Composition of the Body. In: J. Lawrence and C. Tobias (Eds.), Advances in Biological and Medical Physics (Vol. IV). New York, Academic Press.

Thomas, T. and Etheridge, G., 1980 "Hydrostatic weighing at residual volume and functional residual capacity". J.Appl.Physiol. 49: 157-159.

Timson, B. F. and Coffman, J.L., 1984 "Body composition by hydrostatic weighing at total lung capacity and residual volume". Med.Sci.Sports Exerc. 16: 411-414.

Welch, B. and Crisp, C., 1958 "Effect of level of expiration on body density measurement". J.Appl.Physiol. 12: 399-402.

Weltman, A. and Katch, V., 1981 "Comparison of hydrostatic weighing a residual volume and total lung capacity". Med.Sci.Sports Exerc. 13: 210-213.

Wilmore, J., 1969 "The use of actual, predicted, and constant residual volumes in the assessment of body composition by underwate weighing". Med.Sci.Sports 1: 87-90. 